\title{
In vitro methods for specific IgE detection on cow's milk allergy
}

\section{S. Prates, M. Morais-Almeida, V. Matos, V. Loureiro and J. Rosado-Pinto}

Immunoallergy Department. Dona Estefânia Hospital. Lisbon. Portugal.

\section{ABSTRACT}

Background: A new method for determining serum specific lgE (IMMULITE ${ }^{\circledR} 2000$ 3gAllergy) has recently become available.

Objective: To evaluate the clinical performance of IMMULITE 2000 in the diagnosis of cow's milk allergy compared with that of UniCAP ${ }^{\circledR}$. Additionally, we verified the behavior of both methods at two diagnostic decision points proposed by other authors.

Methods: The study population consisted of 31 children with cow's milk allergy (group A) and a control group of 19 atopic children without food allergy (group B). A blood sample from each child was tested using both methods and the results were compared.

Results: In group A, the values for cow's milk IgE ranged from $0.35 \mathrm{kU} / \mathrm{L}$ (the lowest common detection limit) to above $100 \mathrm{kU} / \mathrm{L}$. In group $\mathrm{B}$, the values were less than $1.1 \mathrm{kU} / \mathrm{L}$ for IMMULITE 2000 and less than $1.6 \mathrm{kU} / \mathrm{L}$ for UniCAP. An agreement of $90 \%$ in IgE classes was obtained. Both methods demonstrated exactly the same diagnostic performance (sensitivity: $100 \%$; specificity: $78.9 \%$; negative predictive value: $100 \%$; positive predictive value: $84.6 \%$;

Correspondence:

Sara Prates

Serviço de Imunoalergologia

Hospital Dona Estefânia

Rua Jacinta Marto

1169-045 Lisboa

Portugal

Phone: +351213126653

Fax: +351213126654

E-mail: saraprates@netcabo.pt efficiency: $90.2 \%)$. The evaluation of the two methods at the two different decision points proposed in the literature showed a better positive predictive value with UniCAP, but we obtained equivalent performance with IMMULITE 2000 by choosing higher cutoff values.

Conclusions: We conclude that IMMULITE 2000 is as effective as UniCAP in the diagnosis of cow's milk allergy. Both methods can be used to obtain site-specific decision points that are population, age and disease dependent.

Key words: Cow's milk allergy; food hypersensitivity; immunoassay; infant food; milk proteins; predictive value; specific IgE; IMMULITE ${ }^{\circledR} 2000$ 3gAllergy; UniCAP®

Cow's milk allergy (CMA) occurs mainly during the first years of life and is one of the most prevalent food allergies in many countries. In our country it is by far the most common food allergy in children. An accurate diagnosis is necessary in order to protect children from either inadequate or unnecessary avoidance diets. This usually requires the performance of oral food challenges, exposing the patient to the risk of a severe allergic reaction. Moreover, food challenges are time consuming procedures, especially when they are performed in double blind controlled protocols ${ }^{1,2}$.

Methods for determination of serum allergen specific IgE (slgE) have been improved over the past years and the possibility of quantitative determination of food specific lgE opened new perspectives on the diagnosis and follow-up of these patients. Specific lgE decision points with a high predictive level of 
clinical reactivity to foods like milk, egg, fish and peanut have been established ${ }^{3-5}$. However, the decision points obtained for the same allergens are considerably different, presumably depending on the characteristics of the population studied, such as age and type of clinical manifestations ${ }^{6}$. The method used by these groups was the same - UniCAP ${ }^{\circledR}$ (Pharmacia, Uppsala, Sweden).

A new method for slgE, IMMULITE ${ }^{\circledR} 2000$ 3gAllergy (Diagnostic Products Corporation, Los Angeles, USA) has been introduced recently ${ }^{78}$. In this study, we evaluated the clinical performance of IMMULITE 2000 with respect to detection of cow's milk allergy by comparing it with UniCAP and by verifying, within our population, the behavior of both methods, at two different decision points (cutoffs) as previously proposed by other authors ${ }^{3,4}$.

\section{METHODS}

\section{Patients}

Among the children on follow-up at our outpatient unit for CMA we selected those with indication to be submitted to a follow-up challenge, according to the clinical criteria in use at our department (table I). We included 31 patients (17 males, 14 females) aged between 1 and 9 years old (median: 3,5 years) group A. All cases presented with immediate type reactions-urticaria, angioedema, vomiting or bronchospasm-and all had positive reactions to skin prick tests for whole milk and/or protein fractions (from IPI-ASAC Spain: milk, casein, alpha-lactoalbumin and beta-lactoglobulin). The diagnosis in each had been previously confirmed by positive food challenges.

For the control group (group B), we selected children with atopic dermatitis because this is a situation where it is common to find low levels of food specific

Table I

\begin{tabular}{l}
$\begin{array}{c}\text { Clinical criteria for the performance of follow-up } \\
\text { cow's milk challenge in children with cow's milk allergy }\end{array}$ \\
\hline - First follow-up challenge: \\
- Between 12 and 18 months of age \\
- Subsequent challenges: \\
- 12 months after the last positive challenge \\
(or accidental reaction) for children until 3 years of age \\
- 24 months after the last positive challenge (or accidental \\
reaction) for children between 3 and 6 years of age \\
- 36 months after the last positive challenge (or accidental \\
reaction) for children older than 6 years
\end{tabular}

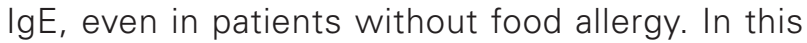
group we included 19 children (10 males, 9 females) aged between 2 and 14 years old (median: 5,0 years), without clinical evidence of CMA, followed by us for other allergic diseases (atopic dermatitis in 19, asthma in 9, rhinitis in 9). The atopic dermatitis was mild and intermitent in all cases, with symptom free periods despite a daily regular intake of dairy products.

\section{Cow's milk oral challenges}

During the study all patients underwent reevaluation, which included an oral milk-challenge test. Informed consent was obtained from the parents, according to the recomendations of the hospital ethics committee. Given the young age of the children and because we expected immediate and objective reactions, we decided to use an open protocol. All challenges were performed under medical surveillance at the day-hospital unit, with appropriate medication and resuscitation equipment available. Increasing doses of cow's milk infant formula $(1,5,10,20,40$, $80 \mathrm{ml}$ with a repeat dose of $80 \mathrm{ml}$, to a cumulative dose of $236 \mathrm{ml}$ ) were given at 30-minute intervals. The challenge was discontinued and considered to be positive if a clinical reaction appeared (urticaria, angioedema, erythematous rash, vomiting, diarrhea, bronchospasm, rhinoconjunctivitis or hypotension). In instances of a clinical reaction, treatment was provided according to the type and severity of symptoms. After the challenge, the child remained under observation for at least six hours. If the challenge was negative, dairy products were freely introduced to the child's diet and a new visit was scheduled within a week to check for the development of late symptoms.

Children from the control group were not challenged since they were regularly consuming dairy products without evidence of an adverse impact on their allergic disease.

\section{In vitro tests}

A blood sample was obtained from each child close to the challenge test-within approximately one month. Each blood sample was tested for cow's milk specific lgE using the IMMULITE $2000^{\circledR}$ and the UniCAP ${ }^{\circledR}$.

IMMULITE 2000 is a third generation slgE twostep chemiluminescent enzyme immunoassay which uses a solid phase (bead). Both the IMMULITE 2000 and UniCAP assays were performed according to the manufacturer's instructions. The results were consid- 
ered positive when a level of, at least, $0.35 \mathrm{kU} / \mathrm{L}$ was obtained.

\section{RESULTS}

During the study, all children in patient group A were rechallenged to assess their state of tolerance. Of 31 milk challenges, 22 were positive (current CMA) and 9 were negative (past CMA). Samples for slgE analysis were taken from all 50 children, (current CMA, past CMA and controls).

The values obtained for milk specific lgE in patient group A ranged from the detection limit of $0.35 \mathrm{kU} / \mathrm{L}$, to the upper limit of $100 \mathrm{kU} / \mathrm{L}$ with $\mathrm{UniCAP}^{\circledR}$ and from below the IMMULITE ${ }^{\circledR} 2000$ detection limit of 0.15 to the upper limit of $100 \mathrm{kU} / \mathrm{L}$. Two patients had negative results $(<0,35 \mathrm{kU} / \mathrm{L})$ with IMMULITE 2000 and three with UniCAP. In the control group B, the values were under $1.1 \mathrm{kU} / \mathrm{L}$ for IMMULITE 2000 and under $1.6 \mathrm{kU} / \mathrm{L}$ for UniCAP. Four patients had low positive results with both methods.

Any value at or below the detection limit of $0.35 \mathrm{kU} / \mathrm{L}$, or at or above the upper limit of $100 \mathrm{kU} / \mathrm{L}$ for either assay, was considered to be out of range. Using these criteria, just 20 of the 50 paired samples fell within the common 0.35 to $100 \mathrm{kU} / \mathrm{L}$ range by both in vitro assays. Working in a logarithmic scale, we compared the results for the 20 in-range samples, noting a trend towards reporting higher concentrations of slgE by the IMMULITE 2000 assay in most patients (fig. 1).

We evaluated the agreement between methods in terms of slgE class (table II). In this case, as the analysis is qualitative, not quantitative, we used data from all 50 children. Considering a class 0 result as negative and classes 1 through 6 as positive, we obtained $90 \%$ agreement with only five discordant results. One of these five belonged to group $A$ and was positive (class 1) according to the IMMULITE 2000 assay, but negative using UniCAP. The challenge test for this patient was negative, indicating that he had achieved clinical remission. The additional four discordant cases were from group B. Two were falsely positive according to UniCAP (class 1) and the other two, using the IMMULITE 2000 (class 2).

We observed good agreement between methods in the lower classes, with results concurring either within the same class or within one class difference. We observed higher results with IMMULITE 2000 in the upper classes; specifically six patients designated as class 6 according to IMMULITE 2000 were designated as class 4 using UniCAP (all were positive by challenge test).

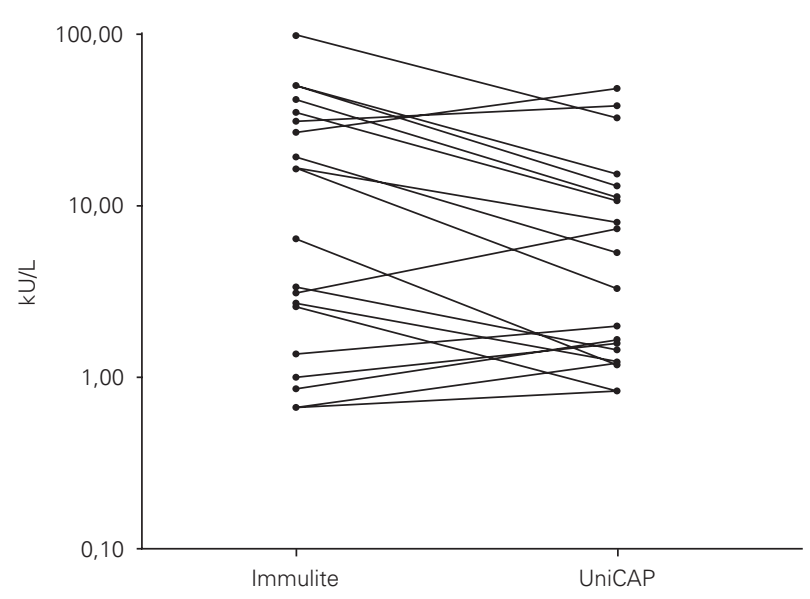

Figure 1.-Cow's milk specific IgE - comparison between methods for the 20 in-range samples (results within 0.35 and $100 \mathrm{kU} / \mathrm{L})$.

Table II

Comparison between methods according to slgE class (90\% agreement)

\begin{tabular}{|c|c|c|c|c|c|c|c|}
\hline \multicolumn{8}{|c|}{ UniCAP } \\
\hline Class & 0 & 1 & 2 & 3 & 4 & 5 & 6 \\
\hline 0 & 15 & 2 & & & & & \\
\hline 8 & 1 & & 2 & & & & \\
\hline$\rightleftarrows$ & 2 & & 5 & 2 & & & \\
\hline$\stackrel{\vec{P}}{P}$ & & & 2 & 1 & & & \\
\hline$\geqq$ & & & & 5 & 2 & & \\
\hline 5 & & & & & 1 & & \\
\hline 6 & & & & & 6 & 3 & 1 \\
\hline
\end{tabular}

To calculate the diagnostic performance of the methods, we included only the 22 patients with active disease (positive challenge) and the 19 controls. Samples were considered positive if values were above $0.35 \mathrm{kU} / \mathrm{L}$ by either method and were compared with the results of the food challenge test. In this sample of 41 patients (CMA prevalence $=53.6 \%$ ), both methods demonstrated the same performance: $100 \%$ sensitivity; $78.9 \%$ specificity; $100 \%$ negative predictive value; $84.6 \%$ positive predictive value; $90.2 \%$ efficiency.

We also evaluated the behavior of both methods at two different decision points previously proposed 
in the literature (Sampson et $\mathrm{al}^{3}$, Garcia-Ara et $\mathrm{al}^{4}$ ). The results also showed a distribution with a fairly clear trend encompassing both of the cutoffs proposed for the Pharmacia system. We applied various symmetric (invertible) regression analyses to the data, using them to predict equivalent cutoffs for the DPC system. Corresponding to the proposed levels of 2.5 and $15 \mathrm{kU} / \mathrm{L}$ for UniCAP, the regression analyses predicted the following levels for IMMULITE 2000: 3.5 and $25 \mathrm{kU} / \mathrm{L}$ by Deming (fig. 2); 3.6 and $33 \mathrm{kU} / \mathrm{L}$ by OLS Bisector (not shown); 5.6 and $44 \mathrm{kU} / \mathrm{L}$ by Passing-Bablok (not shown).

In order to verify how well the two methods discriminated between children with current and past

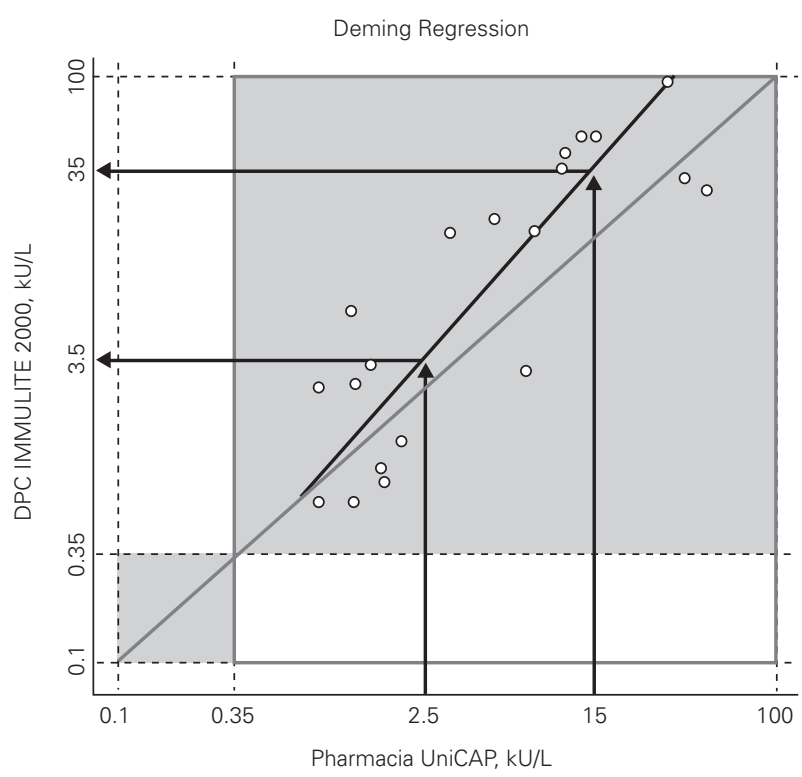

Figure 2.-Prediction of cutoffs for the IMMULITE ${ }^{\circledR} 2000$ 3gAllergy corresponding to published cutoffs for UniCAP ${ }^{\circledR}$ as determined by Deming symmetric regression analyse.

\section{Table III}

\section{Comparison between methods at different cutoffs}

\begin{tabular}{llcccc}
\hline Cutoff & Method & Sensitivity & Specificity & NPV & PPV \\
\hline \multirow{2}{*}{$2,5 \mathrm{kU} / \mathrm{L}$} & IMMULITE 2000 & 95.4 & 55.6 & 83.3 & 84.0 \\
& UniCAP & 90.9 & 88.9 & 80.0 & 95.2 \\
$5 \mathrm{kU} / \mathrm{L}$ & IMMULITE 2000 & 90.9 & 88.9 & 80.0 & 95.2 \\
& UniCAP & 86.4 & 88.9 & 72.7 & 95.0 \\
$15 \mathrm{kU} / \mathrm{L}$ & IMMULITE 2000 & 86.4 & 88.9 & 72.7 & 95.0 \\
& UniCAP & 63.6 & 100.0 & 52.9 & 100.0 \\
$35 \mathrm{kU} / \mathrm{L}$ & IMMULITE 2000 & 63.6 & 100.0 & 52.9 & 100.0 \\
& UniCAP & 36.4 & 100.0 & 39.1 & 100.0 \\
& & & & & \\
\hline
\end{tabular}

NPV: negative predictive value; PPV: positive predictive value.
CMA, we included only those patients in group $A$ within our analysis (table III). At the cutoff decision point of $15 \mathrm{kU} / \mathrm{L}$ (as proposed by Sampson ${ }^{3}$ for milk reactivity) we observed that, in our population, UniCAP shows a slightly better positive predictive value (100\%) than IMMULITE 2000 (95\%), although UniCAP's sensitivity was much lower than IMMULITE's at this cutoff (64\% vs. $86 \%$, respectively). However, we verified that we could obtain exactly the same positive predictive value (100\%) with IMMULITE 2000 by choosing a higher cutoff point of $35 \mathrm{kU} / \mathrm{L}$, albeit at the cost of sensitivity (64\%). Similarly, at the much lower cutoff of $2.5 \mathrm{kU} / \mathrm{L}$ proposed by Garcia$\mathrm{Ara}^{4}$, UniCAP shows a somewhat better positive predictive value than IMMULITE $2000(95.2 \%$ vs. $84.0 \%)$ but once again with lower sensitivity $(90.9 \%$ UniCAP vs. 95.4\% IMMULITE 2000). However, table II demonstrates that the performance and sensitivity of IMMULITE 2000 exactly equals UniCAP's performance if we increase the cutoff to $5 \mathrm{kU} / \mathrm{L}$.

\section{DISCUSSION}

In our study population, IMMULITE ${ }^{\circledR} 2000$ demonstrated reliable results in the detection of cow's milk specific IgE and its performance was found to be comparable to that of UniCAP ${ }^{\circledR}$. IMMULITE 2000 demonstrated a tendency to report results for slgE at greater concentrations, which was more evident in the higher range of results. However, at the usual positive cutoff of $0.35 \mathrm{kU} / \mathrm{L}$, both methods detected all cases of CMA (100\% sensitivity). The specificity was also equal for the two methods and, although somewhat lower $(78.9 \%)$, it was acceptable, considering the particular characteristics of the control group. For this group, we deliberately chose children with atopic dermatitis because this is a clinical situation where we often find high levels of serum IgE, increasing the likelihood of false positive slgE results. With this kind of control group, we found the specificity of the IMMULITE 2000 method was as good as the UniCAP.

The methods of diagnosing IgE mediated food allergy, either by skin prick tests or by assaying for serum slgE, are usually very sensitive but less specific, demanding confirmation of the results by means of oral challenge tests ${ }^{1}$. This has led several groups to try to determine the decision point levels of slgE with a higher positive predictive value for clinical reactivity ${ }^{3-5}$. In a study involving a sample of children with atopic dermatitis, Sampson et al ${ }^{9}$ proposed an optimal decision point of $32 \mathrm{kU} / \mathrm{L}$ for cow's milk allergy. In this population at this decision point level, the test showed a positive predictive value of $95 \%$, a sensi- 
tivity of $51 \%$ and specificity of $98 \%$. In a subsequent work by the same group, this decision point was applied to another population of children referred for evaluation of food allergy, where only $61 \%$ had atopic dermatitis. In this clinically different population, the sensitivity of the test decreased to $34 \%$ and a better performance was obtained by using a lower decision point of $15 \mathrm{kU} / \mathrm{L}$ (57\% sensitivity, $94 \%$ specificity). In a recent prospective study of a group of children with cow's milk allergy, Garcia-Ara et al ${ }^{10}$ showed that the slgE levels, which were predictors of clinical reactivity, increased with age. This suggests that age must also be taken into consideration when establishing slgE cutoffs. All these data strongly suggest that the decision point levels of slgE predictive of clinical reactivity are population specific ${ }^{6}$.

It was not our intention to establish our own cutoff points in this study because our study population was not large enough; rather, we were interested in comparing the behavior of the two methods at the cutoffs established by other authors. We focused on two different decision points, both obtained using the UniCAP, in two different populations. The decision point proposed by Sampson $(15 \mathrm{kU} / \mathrm{L})$ was obtained in a population of older children, most of whom presented with atopic dermatitis. ${ }^{3}$ The other, proposed by Garcia-Ara et al, was somewhat lower $(2.5 \mathrm{kU} / \mathrm{L})$, and was obtained in a population of infants with immediate-type symptoms ${ }^{4}$. The different characteristics of the two populations could explain the different cutoffs obtained by the two groups.

In our study, we obtained the same performance with both methods by using different cutoff levels. Accordingly, the decision points seem also to be specific to the method. The main consequence is that the results obtained by the two methods should not be used interchangeably, since the same result could have a different meaning, depending on the method by which it was obtained.

Our study has shown IMMULITE 2000 3gAllergy to be a reliable method for detecting cow's milk allergy and as adequate as UniCAP in the diagnosis of
CMA. Since decision levels of slgE can depend on population, age, allergens, diseases and in vitro methods, each clinic must define the allergen cutoff for their own population. Care must be taken in avoiding a generalization of the use of the published cutoffs. The comparable clinical performance for milk allergy reported in this study indicated that either of the two methods can be used to obtain the population, age, disease and site-specific decision cutoff levels.

\section{REFERENCES}

1. Sicherer SH. Food allergy: when and how to perform oral food challenges. Pediatr Allergy Immunol 1999;10:226-34.

2. Bindslev-Jensen C, Ballmer-Weber BK, Bengtsson U, Blanco C, Ebner C, Hourrihane J, et al. Standardization of food challenges in patients with immediate reactions to foods - position paper from the European Academy of Allergology and Clinical Immunology. Allergy 2004;59:690-7.

3. Sampson HA. Utility of food specific lgE concentrations in predicting symptomatic food allergy. J Allergy Clin Immunol 2001;107:891-6.

4. García-Ara C, Boyano-Martínez T, Díaz-Pena JM, Martín-Muñoz F, Reche-Frutos M, Martín-Esteban M. Specific IgE levels in the diagnosis of immediate hypersensitivity to cow's milk protein in the infant. J Allergy Clin Immunol 2001;107:185-90.

5. Boyano-Martinez T, García-Ara C, Diaz-Pena JM, Martin-Muñoz F, Garcia-Sanchez G, Martin-Esteban M. Validity of specific $\lg E$ antibodies in children with egg allergy. Clin Exp Allergy 2001;31:1464-9.

6. Malandain $\mathrm{H}$. Are quantitative results of serum specific lgE assays clinically meaningful? Immuno-analyse Biologie spécialisée 2003;18:144-51.

7. Li TM, Chuang T, Tse S, Hovanec-Burns D, El Shami AS. Development and validation of a third generation allergen-specific IgE assay on the continuous random access IMMULITE ${ }^{\circledR}$ 2000 analyzer. Ann Clin Lab Sci 2004;34:67-74.

8. Guilloux L, Hamberger C. Assessment of specific IgE assay using IMMULITE ${ }^{\circledR} 2000$ DPC. Immuno-analyse Biologie spécialisée 2004;19:71-80.

9. Sampson HA, Ho DG. Relationship between food specific lgE concentrations and the risk of positive food challenges in children and adolescents. J Allergy Clin Immunol 1997;100:444-51.

10. Garcia-Ara MC, Boyano-Martinez MT, Diaz-Pena JM, MartinMunoz MF, Martin-Esteban M. Cow's milk specific immunoglobulin $E$ levels as predictors of clinical reactivity in the follow-up of the cow's milk allergy infants. Clin Exp Allergy 2004;34: 866-70. 